\title{
First germanium-based constraints on sub-MeV Dark Matter with the EDELWEISS experiment
}

\author{
Q. Arnaud, ${ }^{1,}$ | E. Armengaud,${ }^{2}$ C. Augier,${ }^{1}$ A. Benoît,${ }^{3}$ L. Bergé ${ }^{4}$ J. Billard,${ }^{1}$ A. Broniatowski, ${ }^{4}$ P. Camus, ${ }^{3}$ \\ A. Cazes, ${ }^{1}$ M. Chapellier, ${ }^{4}$ F. Charlieux, ${ }^{1}$ M. De Jésus, ${ }^{1}$ L. Dumoulin,${ }^{4}$ K. Eitel,${ }^{5}$ E. Elkhoury, ${ }^{1}$ J.-B. Fillipini, ${ }^{1}$ \\ D. Filosofov, ${ }^{6}$ J. Gascon, ${ }^{1}$ A. Giuliani, ${ }^{4}$ M. Gros,${ }^{2}$ Y. Jin, ${ }^{7}$ A. Juillard,${ }^{1}$ M. Kleifges, ${ }^{8}$ H. Lattaud, ${ }^{1}$ S. Marnieros,${ }^{4}$ \\ D. Misiak,${ }^{1}$ X.-F. Navick, ${ }^{2}$ C. Nones, ${ }^{2}$ E. Olivieri,${ }^{4}$ C. Oriol,${ }^{4}$ P. Pari, ${ }^{9}$ B. Paul,${ }^{2}$ D. Poda,${ }^{4}$ S. Rozov,${ }^{6}$ \\ T. Salagnac, ${ }^{1}$ V. Sanglard, ${ }^{1}$ B. Siebenborn,${ }^{5}$ L. Vagneron, ${ }^{1}$ M. Weber,${ }^{8}$ E. Yakushev,${ }^{6}$ and A. Zolotarova ${ }^{4}$
}

(EDELWEISS Collaboration)

\author{
${ }^{1}$ Univ Lyon, Université Lyon 1, CNRS/IN2P3, IP2I-Lyon, F-69622, Villeurbanne, France \\ ${ }^{2}$ IRFU, CEA, Université Paris-Saclay, F-91191 Gif-sur-Yvette, France \\ ${ }^{3}$ Institut Néel, CNRS/UJF, 25 rue des Martyrs, BP 166, 38042 Grenoble, France \\ ${ }^{4}$ Université Paris-Saclay, CNRS/IN2P3, IJCLab, 91405 Orsay, France \\ ${ }^{5}$ Karlsruher Institut für Technologie, Institut für Kernphysik, Postfach 3640, 76021 Karlsruhe, Germany \\ ${ }^{6}$ JINR, Laboratory of Nuclear Problems, Joliot-Curie 6, \\ 141980 Dubna, Moscow Region, Russian Federation \\ ${ }^{7}$ C2N, CNRS, Univ. Paris-Sud, Univ. Paris-Saclay, 91120 Palaiseau, France \\ ${ }^{8}$ Karlsruher Institut für Technologie, Institut für Prozessdatenverarbeitung \\ und Elektronik, Postfach 3640, 76021 Karlsruhe, Germany \\ ${ }^{9}$ IRAMIS, CEA, Université Paris-Saclay, F-91191 Gif-sur-Yvette, France
}

(Dated: March 11, 2022)

\begin{abstract}
The EDELWEISS collaboration has performed a search for Dark Matter (DM) particles interacting with electrons using a $33.4 \mathrm{~g}$ Ge cryogenic detector operated underground at the LSM. A charge resolution of 0.53 electron-hole pairs (RMS) has been achieved using the Neganov-Trofimov-Luke amplification with a bias of $78 \mathrm{~V}$. We set the first Ge-based constraints on sub-MeV $/ \mathrm{c}^{2} \mathrm{DM}$ particles interacting with electrons, as well as on dark photons down to $1 \mathrm{eV} / \mathrm{c}^{2}$. These are competitive with other searches. In particular, new limits are set on the kinetic mixing of dark photon DM in a so far unconstrained parameter space region in the 6 to $9 \mathrm{eV} / \mathrm{c}^{2}$ mass range. These results demonstrate the high relevance of cryogenic Ge detectors for the search of DM interactions producing eV-scale electron signals.
\end{abstract}

PACS numbers: 95.35.+d; 95.85.Pw

Direct-detection experiments are progressing rapidly in the search of nuclear scattering events due to Weakly Interacting Massive Particles on the $\mathrm{GeV} / \mathrm{c}^{2}$ to $\mathrm{TeV} / \mathrm{c}^{2}$ mass scale 1-4. However, there are compelling models that motivate to extend direct searches to Dark Matter $(\mathrm{DM})$ particles in the $\mathrm{eV} / \mathrm{c}^{2}$ to $\mathrm{MeV} / \mathrm{c}^{2}$ range, where the signal would be an electron recoil arising either from the absorption of a dark photon (bosonic DM) [5, 6, or the elastic scattering of a dark fermion [7. For these searches - requiring kg-scale detectors with $\sim 1 \mathrm{eV}$ detection thresholds to fully cover benchmark models [8] semiconductor detectors are uniquely positioned due to their band-gap energies an order of magnitude lower than the ionization potential of xenon-based detectors [9].

Recent progress has been made with silicon-based gram-scale devices, using CCDs [10, 11] and cryogenic detectors [12] now sensitive to single electron-hole pairs. Efforts are ongoing to reduce dark currents and radioactive background to the levels required for scaling up to more massive arrays. In this context, phononmediated germanium detectors offer an attractive alter-

*Electronic address: q.arnaud@ipnl.in2p3.fr native. The smaller band-gap energy of Ge relative to $\mathrm{Si}$ $\left(E_{g}=0.67 \mathrm{eV}\right.$ vs. $\left.1.11 \mathrm{eV}[13,14]\right)$ naturally yields an increased sensitivity to lighter DM particles. In addition, the difference in composition paves the way to a better understanding of the origin of the background observed in semiconductor detectors at this new $\mathrm{eV}$-scale frontier.

In phonon-mediated cryogenic detectors, the drift of $N$ electron-hole pairs across a voltage difference $\Delta V$ produces additional phonons whose energy $E_{\mathrm{NTL}}=N \Delta V$ (in $\mathrm{eV}$ ) adds up to the initial recoil energy. This effect called Neganov-Trofimov-Luke (NTL) [15, 16] essentially turns a cryogenic calorimeter (operated at $\Delta V=0 \mathrm{~V}$ ) into a charge amplifier of mean gain $\langle g\rangle=(1+\Delta V / \epsilon)$, where $\epsilon=3.0 \mathrm{eV}(3.8 \mathrm{eV})$ is the mean ionization energy in $\mathrm{Ge}(\mathrm{Si})$ [17] for electron recoils.

Recently, the EDELWEISS collaboration achieved a $17.7 \mathrm{eV}$ phonon baseline resolution (RMS) with a $33.4 \mathrm{~g}$ Ge bolometer operated above-ground [18. To reach sub-electron-hole pair resolution, a similar detector was equipped with electrodes to take advantage of the expected $1 /\langle g\rangle$ improvement of the charge resolution with applied voltage. In this letter, we exploit the resulting sensitivity to energy deposits as low as the band-gap energy to set competitive constraints on sub-MeV/ $\mathrm{c}^{2} \mathrm{DM}$ particles interacting with electrons, as well as on dark 
photons down to $1 \mathrm{eV} / \mathrm{c}^{2}$.

The DM search was performed at the Laboratoire Souterrain de Modane (France) with a detector consisting of a $33.4 \mathrm{~g}$ cylindrical high-purity Ge crystal $(\oslash 20 \times 20 \mathrm{~mm})$. Aluminum electrodes are lithographed on both planar surfaces in a grid scheme, except for the outer edges where they are shaped as concentric guard rings. A $2 \times 2 \mathrm{~mm}^{2}$ area was left empty at the center of one face to allow for the direct gluing of a Ge neutrontransmutation-doped (NTD) 19 thermal sensor on the crystal. The center and guard electrodes on the same side are biased to the same voltage, but the associated ionization channels are read out separately. The data acquisition system and readout electronics are the same as in 20]. The data from the phonon and ionization channels were digitized at a frequency of $100 \mathrm{kHz}$, filtered, averaged and continuously stored on disk with a digitization rate of $500 \mathrm{~Hz}$. For the DM search, only the phonon channel is used.

The detector was maintained at a regulated temperature of either 20.0 or $20.7 \mathrm{mK}$ between January and October 2019. Most of that period was devoted to detector studies and calibrations. Prior to its installation in the cryostat, the detector was uniformly activated using a neutron AmBe source. The produced short-lived isotope ${ }^{71} \mathrm{Ge}$ decays by electron capture in the K, L and M shells, emitting characteristic lines at 10.37, 1.30 and $0.16 \mathrm{keV}$, respectively. The activation lines are locally absorbed, thus providing very good probes of the detector response to a DM signal uniformly distributed inside the detector volume. These are clearly visible in Fig. 1, which shows the energy spectrum - in units of eV-electron-equivalent $\left(\mathrm{eV}_{\mathrm{ee}}\right)$ - from calibration data recorded in January at biases of $66 \mathrm{~V}$ and $70 \mathrm{~V}$. The measured L/K and M/L yields of $0.110 \pm 0.008$ and $0.158 \pm 0.020$ are compatible with existing measurements [21, 22]. The resolution on the $160 \mathrm{eV}$ peak of $\sigma=8 \mathrm{eV}_{\text {ee }}$ is consistent with the Fano factor $\mathrm{F}=0.15$ expected for $\mathrm{Ge}$ at low energy 23. The precision on the $\mathrm{K}$-line position is better than $0.1 \%$. By varying the bias from $0 \mathrm{~V}$ to $81 \mathrm{~V}$, the non-linearity of the heat sensor signal was measured to be less than $5 \%$ over three orders of magnitude, with a $2 \%$ uncertainty extrapolation down to zero energy.

The $\mathrm{K}$ and $\mathrm{L}$ peaks are accompanied by a low-energy tail of events. On the basis of the corresponding signals observed on the center and guard electrodes, these tails are ascribed to incomplete charge collection for events near the cylindrical surfaces. To prevent charge build-up that would otherwise worsen the collection performance, the detector was regularly grounded for periods of $2-10 \mathrm{~h}$ while being exposed to a strong ${ }^{60}$ Co source. This regeneration procedure allows us to neutralize residual fields induced by the accumulation of trapped charges [20]. The tail represents $19 \%$ of the $\mathrm{K}$ line events above $1.5 \mathrm{keV}_{\text {ee }}$ in Fig. 1. No significant increase of that tail is observed in the days following a regeneration, with an upper limit of $+1 \%$ per day.

A bias of up to $81 \mathrm{~V}$ could be applied without heat-

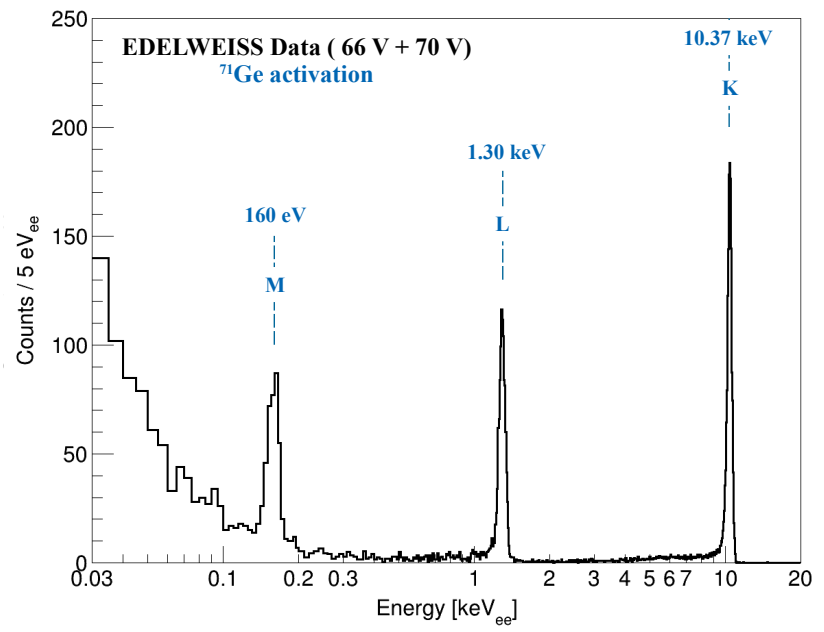

FIG. 1: Energy spectrum recorded with a bias of $66 \mathrm{~V}$ and $70 \mathrm{~V}$ following the ${ }^{71} \mathrm{Ge}$ activation of the detector.

ing up the detector. Ramping up the bias produces an additional noise on the phonon channel. Most of it ebbs away after a period of $12-72 \mathrm{~h}$, after which the baseline resolution at $78 \mathrm{~V}$ is typically $10 \%$ above its value at $\sim 0 \mathrm{~V}$, once the NTL amplification is taken into account. Attempts to use the "pre-biasing" method [12] did not significantly reduce this period.

The study of the detector performance and stability led to the choice of a bias of $78 \mathrm{~V}$ for a DM search involving electron recoils. A continuous sequence of runs at $78 \mathrm{~V}$ from April $1^{\text {st }}$ to $7^{\text {th }}$ were set aside for this search. The baseline resolution derived from random trigger samples was studied hour by hour. The first three days were discarded as the baseline resolution reached its plateau only at the end of that period. The remaining $89 \mathrm{~h}$ of data were separated into a blind sample of $58 \mathrm{~h}$ sandwiched between a non-blind sample of $21 \mathrm{~h}$ plus $9 \mathrm{~h}$ of data. The stability of the energy scale was monitored day by day using the K-line peak. The average baseline energy resolution in the non-blind sample is $1.63 \mathrm{eV}_{\mathrm{ee}}$ (0.54 electron-hole pairs), corresponding to a phonon resolution of $44 \mathrm{eV}$, once the NTL gain of 27 is considered. The average baseline resolution in the blind sample is $3 \%$ better (1.58 $\mathrm{eV}_{\mathrm{ee}}$, or 0.53 pairs). Three days after the data taking described above, the detector was exposed again to a strong AmBe source for $15 \mathrm{~h}$, in order to reactivate it and confirm the stability of the detector response with high statistics.

The data processing - based on an optimal matching filter approach - is essentially the same as in [18 and uses the numerical procedure described in [24. An iterative search for pulses in the filtered data stream is performed using a decreasing energy ordering rule. After the pulse with the largest amplitude is found, a time trace of $\Delta t=2.048 \mathrm{~s}$ is allocated. This period is excluded from the search in the next iteration, proceeding downward in amplitude. The procedure stops when there is no time interval greater than $\Delta t$ left in the stream. Thus, there 
is no trigger threshold set in energy and the trigger rate is driven by the choice of $\Delta t$, not by the physical event rate. The energy dependence of the dead time induced by this procedure is fully taken into account in the evaluation of the trigger efficiency using a pulse simulation described below.

The pulse amplitudes are evaluated by minimizing a $\chi_{k}^{2}$ function in the frequency domain. The subscript $k$ designates the so-called "normal" and "fast" categories of events, each corresponding to a different pulse template. Normal events refer to particle interactions occurring in the Ge target crystal. For this category, we use a template based on $10.37 \mathrm{keV}$ event pulses which are characterized by a rise time of $\sim 7 \mathrm{~ms}$. Fast events stand out with a considerably shorter rise time ( $<1 \mathrm{~ms})$, compatible with interactions occurring directly in the NTD. The data selection is based on the values of $\chi_{\text {normal }}^{2}$ and on the difference $\Delta \chi^{2}=\chi_{\text {normal }}^{2}-\chi_{\text {fast }}^{2}$ whereas pulse amplitude estimation is based on the normal template only.

The trigger and cut efficiencies were determined using a complete signal simulation procedure [18]. Pulses of known energy are injected at random times throughout the entire real data streams at a rate of $\sim 0.02 \mathrm{~Hz}$ in order not to increase the deadtime by more than $\sim 1 \%$. Each simulated pulse corresponds to a trace randomly chosen among a selection of K-line events, scaled to the desired fraction of $10.37 \mathrm{keV}$ and added to the data stream. The set of preselected traces consists of 858 $\mathrm{K}$-line events with energies between 1.5 and $11 \mathrm{keV}_{\text {ee, }}$ recorded at $78 \mathrm{~V}$ after post-search activation. The effect of incomplete charge collection on the signal detection efficiency is conservatively accounted for by ascribing a $0 \%$ survival probability to simulated pulses from traces of $\mathrm{K}$ line events with an energy $470 \mathrm{eV}_{\mathrm{ee}}(\sim 3 \sigma)$ away from the $10.37 \mathrm{keV}_{\text {ee }}$ peak. This results in a $25 \%$ efficiency $\operatorname{loss}^{1}$ visible in Fig. 2 showing the trigger and analysis cut efficiencies as a function of the injected pulse energy. The fraction of simulated events surviving the reconstruction procedure and the selection cut on the value of $\chi_{\text {normal }}^{2}$ are shown as orange line. The plateau reduction from $75 \%$ to $65 \%$ is due in large part to losses due to reset periods required for the operation of the charge readout. The decrease of efficiency at low energy is due to the trigger algorithm bias toward high-energy events. The cut on $\Delta \chi^{2}$ further reduces the plateau to $59 \%$ at $30 \mathrm{eV}_{\mathrm{ee}}$. After all cuts the efficiency for simulated single (double) electron-hole pair events of $3 \mathrm{eV}_{\mathrm{ee}}\left(6 \mathrm{eV}_{\mathrm{ee}}\right)$ is $4 \%(22 \%)$. Fig. 3. shows the energy spectrum of the selected events in the 58 hours of DM search. The efficiency-corrected rate at $25 \mathrm{eV}_{\text {ee }}$ corresponds to $1.6 \times 10^{5}$ events $/ \mathrm{kg} / \mathrm{day} / \mathrm{keV}_{\text {ee }}$. Comparison of the spectra recorded at different biases

\footnotetext{
1 This is larger than the $19 \%$ tail fraction from Fig. 1 due to the reduced $\mathrm{K}$-line event rate ( 3 vs. $15 \mathrm{mHz}$ ) in the post-search activation sample compared to a constant Compton background.
}

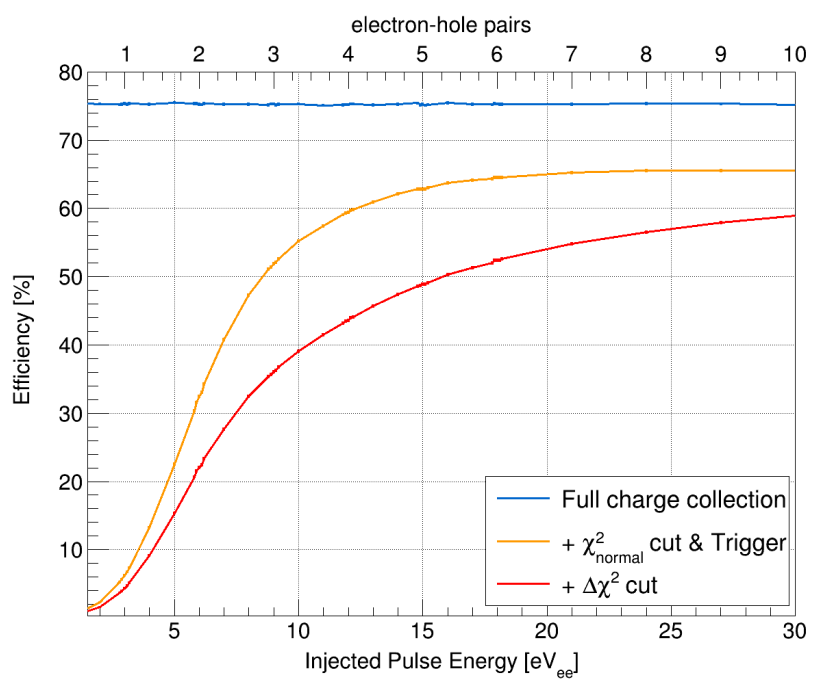

FIG. 2: Signal efficiency measured as the fraction of pulses injected in the data stream that pass successive selection criteria, as a function of the input phonon energy.

suggests that most of the rate observed above $25 \mathrm{eV}_{\mathrm{ee}}$ at $78 \mathrm{~V}(0.68 \mathrm{keV}$ total phonon energy) corresponds to events not affected by the NTL amplification. The origin of these so-called "heat-only" events is still under investigation $^{2}$. Although it limits the detection of electron recoils above $\sim 10 \mathrm{eV}_{\text {ee }}$, the main limitation for signals associated to fully-collected 1 to 3 electron-hole pairs is the rapid rise of the spectrum at low energy. In Fig. 3 , we show the contributions of $N=[1, \ldots, 5]$ electron-hole pair events obtained from the pulse simulation for models described below. For $N>1$, the reconstructed energy spectra associated to $N$-pair events peak at $N \times \epsilon \mathrm{eV}_{\text {ee }}$. However, the spectrum associated to single-pair events is biased towards higher energy as only those with reconstructed energies above $\sim 3 \mathrm{eV}$ are selected by the trigger algorithm. The detector resolution is not sufficient to unambiguously disentangle single-pair from noise-triggered events. It is however able to provide an upper bound on single-pair (or $N$-pair events), and more generally to the DM signals discussed below.

The DM-electron scattering rate as a function of the energy transfered to the electron $E_{e}$ is given by [7]:

$$
\frac{d N}{d E_{e}} \propto \bar{\sigma}_{e} \int \frac{d q}{q^{2}} \eta\left(E_{e}, q, m_{\chi}\right)\left|F_{D M}(q)\right|^{2}\left|f_{c}\left(q, E_{e}\right)\right|^{2},
$$

where $\bar{\sigma}_{e}$ is a reference cross section for free electron scattering. The term $\eta$ encapsulates DM halo physics and is calculated assuming a local DM density of $\rho=0.3 \mathrm{GeV} / \mathrm{c}^{2} / \mathrm{cm}^{3}$, a galactic escape velocity

\footnotetext{
2 The rate observed at $25 \mathrm{eV}_{e e}$ would correspond to a background of $6 \times 10^{3}$ events $/ \mathrm{kg} / \mathrm{day} / \mathrm{keV}$ at $0.68 \mathrm{keV}$ if these are assumed to have no associated charge. This is a factor 3 below the background observed above ground at this energy in [18].
} 


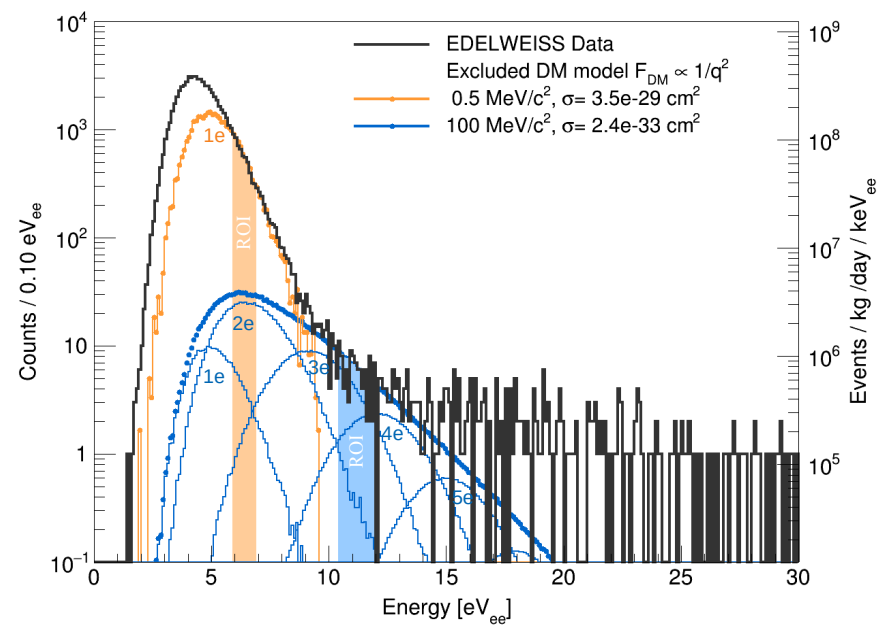

FIG. 3: Energy spectrum of the events selected for the DM search (black). The thick blue (orange) histogram is the simulation of the signal excluded at $90 \%$ C.L. for a DM particle with a mass of $10(0.5) \mathrm{MeV} / \mathrm{c}^{2}$, and $F_{D M}=1 / q^{2}$. The thin-line histograms of the same color represent the individual contributions of 1 to 5 electron-hole pairs. The corresponding ROIs used to set the upper limits are shown as shaded intervals using the same color code.

$\mathrm{v}_{\mathrm{esc}}=544 \mathrm{~km} / \mathrm{s}$ and an asymptotic circular velocity $\mathrm{v}_{0}=$ $220 \mathrm{~km} / \mathrm{s}[25$, 26. The momentum-transfer $q$ dependence of the interaction is described by the form factor $F_{D M}$. The crystal form factor $f_{c}$ is related to the probability that a momentum transfer $q$ yields an electron transition of energy $E_{e}$, given the details of the Ge crystal band structure. It is computed with the QEdark module [7] of the Quantum ESPRESSO package [27.

For the search of a dark photon, its absorption rate per unit time and target mass is calculated according to [6]

$$
R=\frac{1}{\rho} \frac{\rho_{D M}}{m_{V}} \kappa_{\mathrm{eff}}^{2}\left(m_{V}, \tilde{\sigma}\right) \sigma_{1}\left(m_{V}\right),
$$

where $m_{V}$ is the dark photon mass and the expected signal is a mono-energetic electron transition of energy $E_{e}=m_{V} c^{2} . \kappa_{\text {eff }}$ is the effective mixing angle which is linearly proportional to the kinetic mixing parameter $\kappa$ between the Standard Model (SM) photon and its hidden counterpart, and $\sigma_{1}$ is the real part of the complex conductivity $\tilde{\sigma}$. In Ge, the temperature dependence of $\tilde{\sigma}$ above $1 \mathrm{eV}$ is small, allowing us to use the room temperature data from [6] down to $1 \mathrm{eV} / \mathrm{c}^{2}$.

The signal recorded in the detector, calibrated in $\mathrm{eV}_{\mathrm{ee}}$, is $E=\left(E_{e}+N \Delta V / \epsilon\right) /(1+\Delta V / \epsilon)$, thus requiring a discrete distribution function to ascribe a probability $P\left(N \mid E_{e}\right)$ of producing $N$ electron-hole pairs following an electron transition of energy $E_{e}$. A variety of ionization models have been proposed [7, 12, 28. Here, we use the ionization model of [12] (with $\mathrm{F}=0.15$ ) in order to facilitate the comparison of our results with those obtained with this Si phonon-mediated detector.
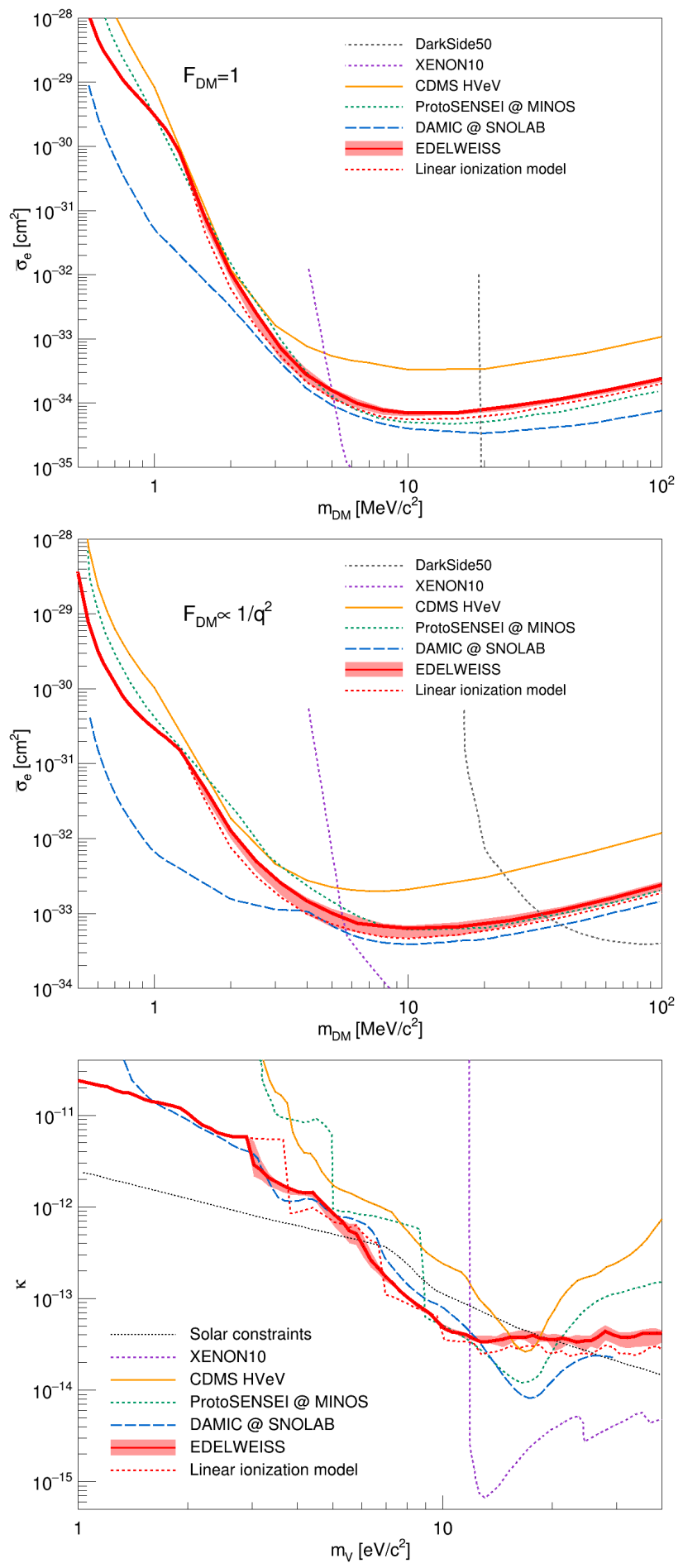

FIG. 4: $90 \%$ C.L. upper limit on the cross section for the scattering of DM particles on electrons, assuming a heavy (top panel) or light (middle panel) mediator. Bottom: 90\% C.L. upper limit on the kinetic mixing $\kappa$ of a dark photon. The results from the present work are shown as the red line. The shaded red band and dotted red line represent alternative charge distribution models (see text). Also shown are constraints from other direct detection experiments [7, 9, 12, 29, and solar constraints 30, 31. 
The method to derive $90 \%$ C.L. upper limits on the DM interaction and absorption rates from the observed spectrum is the same as in [18. DM-mass dependent regions of interest (ROIs) are defined on the non-blind sample prior to the unblinding of the search sample. These ROIs are chosen such as to optimize the signal-to-background ratio between the simulated signals and the $30 \mathrm{~h}$ of nonblind data. In order to reduce the importance of statistical fluctuations in the non-blind data set, its energy spectrum was smoothed with a kernel density estimator and a minimum width of $1 \mathrm{eV}$ was imposed to the ROIs. Once these ROIs are defined for the different DM models and mass values, $90 \%$ C.L. upper limits on their interaction rates are derived using Poisson statistics, considering all events in the search sample ROIs as DM candidates. Because no background subtraction is performed, this procedure yields conservative bounds even if a signal is present in the non-blind data set as it would only result in the ROIs being non optimized.

These limits are shown as the solid red lines in Fig. 4. The top and middle panels are the limits for the interaction cross-section $\bar{\sigma}_{e}$ of DM particles with electrons via a heavy $\left(F_{\mathrm{DM}}=1\right)$ or light mediator $\left(F_{\mathrm{DM}} \propto 1 / q^{2}\right)$, respectively. The bottom panel shows the limits on the kinetic mixing parameter $\kappa$ of a dark photon with a SM one. Variation on the energy scale of $\pm 2 \%$ would affect the limits on $\kappa\left(\bar{\sigma}_{e}\right)$ by less than $10 \%(20 \%)$. Temperature effects on $\sigma_{1}$ in Ge for electron signals above $1 \mathrm{eV}_{\text {ee }}$ are expected to be small: it was nevertheless verified that a $20 \%$ variation of $\sigma_{1}$ would affect the limits on $\kappa$ by at most $\pm 10 \%$. The excluded event rates are at levels where Earth-shielding effects are negligible 32].

The light red band shows the effect of varying the Fano factor $\mathrm{F}$ between 0.30 and the lower bound set by the Bernoulli distribution [28]. The dotted red lines are the limits derived using the linear ionization model described in [7, whereby the $P\left(N \mid E_{e}\right)$ distribution is replaced by a delta function at the floor value of $N=1+\left(E_{e}-E_{g}\right) / \epsilon$. This results in a step-wise evolution of the limits on $\kappa$ as a function of $m_{V}$, as the sensitivity for a given mass is entirely based on the limit on the rate of $N$-pair events. The noticeable difference around $m_{V}=3 \mathrm{eV} / \mathrm{c}^{2}$ between the dark photon limits obtained when considering these two different ionization models is due to the minimum energy needed to create two-pair events $\left(\epsilon\right.$ vs. $\left.\epsilon+E_{g}\right)$. The EDELWEISS sensitivity below $m_{V}=3 \mathrm{eV} / \mathrm{c}^{2}$ derives from a $90 \%$ C.L. upper bound of $4 \mathrm{~Hz}$ on the efficiencycorrected rate of single-pair events in the detector. The upper limit on the two-pair event rate is $0.08 \mathrm{~Hz}$. The single-electron rate corresponds to a contribution to the leakage current of the detector of $<6.4 \times 10^{-19} \mathrm{~A}$.

The present DM constraints extend to much smaller masses than searches based on noble gas detectors 9, 29 and are competitive with those obtained with Si-based detectors [10 12]. In particular, the present limits are the most stringent ones on the kinetic mixing parameter $\kappa$ for dark photon masses between 6 and $9 \mathrm{eV} / \mathrm{c}^{2}$. The better sensitivity of Ge compared to Si for $m_{V}=1 \mathrm{eV} / \mathrm{c}^{2}$ is due to the difference in gap energies. In this respect, $\mathrm{Ge}$ is a more favorable target for low-mass dark photon searches. For DM-electron scattering above $1 \mathrm{MeV} / \mathrm{c}^{2}$, Si benefits from more favorable values of $f_{c}$.

The improvement by an order of magnitude of the detection threshold for electron recoils compared to [18 provides important constraints to understand the origin of the background limiting low-mass DM searches. Further progress in resolving the contributions of heat-only and single-pair events should come from an improvement of the energy resolution.

In the context of the EDELWEISS-SubGeV program, this will be achieved by upgrading the front-end electronics 33 and by operating the NTD sensor at lower temperature to improve its sensitivity. To improve the resolution after NTL amplification, the collaboration studies methods to better control the noise induced at large biases and develops detectors with alternative electrode schemes, such as double-sided vacuum electrodes. The collaboration also investigates sensors based on Superconducting Single-Photon Detectors [34, 35] as a possible way to tag ionizing events down to a single charge and thus provide a very efficient rejection of any heat-only background.

In conclusion, the results obtained demonstrate for the first time the high relevance of cryogenic Ge detectors for the search of DM interactions producing eV-scale electron signals and represent an important milestone of the EDELWEISS-SubGeV program which aims at further probing a variety of DM models in the $\mathrm{eV} / \mathrm{c}^{2}$ to $\mathrm{GeV} / \mathrm{c}^{2}$ mass range.

\section{Acknowledgments}

The help of the technical staff of the Laboratoire Souterrain de Modane and the participant laboratories is gratefully acknowledged. The EDELWEISS project is supported in part by the German Helmholtz Alliance for Astroparticle Physics (HAP), by the French Agence Nationale pour la Recherche (ANR) and the LabEx Lyon Institute of Origins (ANR-10-LABX-0066) of the Université de Lyon within the program "Investissements d'Avenir" (ANR-11-IDEX-00007), by the P2IO LabEx (ANR-10-LABX-0038) in the framework "Investissements d'Avenir" (ANR-11-IDEX-0003-01) managed by the ANR (France), and the Russian Foundation for Basic Research (grant No. 18-02-00159). This project has received funding from the European Union's Horizon 2020 research and innovation programme under the Marie Skłodowska-Curie Grant Agreement No. 838537. We thank J.P. Lopez (IP2I), F. Larger, S. Tabtou (Labrador platform IP2I) and the Physics Department of Université Lyon 1 for their contribution to the radioactive sources. 
[1] E. Aprile et al. (XENON Collaboration), Phys. Rev. Lett. 121, 111302 (2018), arXiv:1805.12562

[2] D.S. Akerib et al. (LUX Collaboration), Phys. Rev. Lett. 118, 021303 (2017) arXiv:1608.07648.

[3] A. Tan et al. (PandaX-II Collaboration), Phys. Rev. Lett. 117, 121303 (2016) arXiv:1607.07400

[4] P. Agnes et al. (DarkSide Collaboration), Phys. Rev. Lett. 121, 081307 (2018) arXiv:1802.06994

[5] H. An, M. Pospelov, J. Pradler, and A. Ritz, Phys. Lett. B747, 331 (2015) arXiv:1412.8378

[6] Y. Hochberg, T. Lin, and K.M. Zurek, Phys. Rev. D 95, 023013 (2017) arXiv:1608.01994

[7] R. Essig et al., J. High Energ. Phys. 2016, 46 (2016) arXiv:1509.01598

[8] M. Battaglieri et al., US Cosmic Visions: New Ideas in Dark Matter 2017: Community Report, arXiv:1707.04591

[9] I.M. Bloch et al., J. High Energ. Phys. 2017, 87 (2017) arXiv:1608.02123

[10] O. Abramoff et al. (SENSEI Collaboration), Phys. Rev. Lett. 122, 161801 (2019) arXiv:1901.10478

[11] A. Aguilar-Arevalo et al. (DAMIC Collaboration), Phys. Rev. Lett. 123, 181802 (2019) arXiv:1907.12628

[12] R. Agnese et al. (CDMS Collaboration), Phys. Rev. Lett. 121, 051301 (2018) Erratum: Phys. Rev. Lett. 122, 069901 (2019) arXiv:1804.10697

[13] B. G. S. B. Streetman, Solid state electronic devices, Prentice Hall (2005)

[14] C.A. Klein, J. Appl. Phys. 39, 2029 (1968)

[15] P.N. Luke, J. Appl. Phys. 64, 6858 (1988).

[16] B.S. Neganov and V.N. Trofimov, Otkrytia i Izobret. 146, 215 (1985), USSR Patent No. 1037771.

[17] G.F. Knoll, Radiation Detection and Measurement, Fourth Edition, New York: John Wiley and Sons (2010)
[18] E. Armengaud et al. (EDELWEISS Collaboration), Phys. Rev. D 99, 082003 (2019) arXiv:1901.03588

[19] E.E. Haller et al., SPIE 2198, Instrumentation in Astronomy VIII (1994) 630

[20] E. Armengaud et al. (EDELWEISS Collaboration), JINST 12, P08010 (2017), arXiv:1706.01070.

[21] J.N. Bahcall, Phys. Rev. 132, 362 (1963).

[22] H. Genz, J.P. Renier, J.G. Pengra and R.W. Fink, Phys. Rev. C 3, 172 (1971).

[23] B.G. Lowe, Nucl. Instrum. Meth. A 399, 354 (1997)

[24] S. Di Domizio, F. Orio and M. Vignati, JINST 6, P02007 (2011), arXiv:1012.1263

[25] J.D. Lewin and P.F. Smith, Astropart. Phys. 6, 87 (1996)

[26] C. Savage, K. Freese and P. Gondolo, Phys. Rev. D 74, 043531 (2006), astro-ph/0607121.

[27] P. Giannozziet et al., Journal of physics: Condensed matter 21, 395502 (2009) arXiv:0906.2569

[28] D. Durnford, Q. Arnaud and G. Gerbier, Phys. Rev. D 98, 103013 (2018) arXiv:1808.06967

[29] P. Agnes et al, Phys. Rev. Lett. 121, 111303 (2018) arXiv:1802.06998

[30] H. An, M. Pospelov, and J. Pradler, Phys. Lett. B 725, 190 (2013) arXiv:1302.3884

[31] J. Redondo and G. Raffelt, JCAP 1308, 034 (2013) arXiv: 1305.2920

[32] T. Emken, R. Essig, C. Kouvaris and M. Sholapurkar, JCAP 09 (2019) 070. arXiv:1905.06348

[33] A. Juillard et al., J. Low Temp. Phys. (2019) arXiv:1909.02879

[34] G.N. Goltsman et al., Appl. Phys. Lett. 79, 705 (2001)

[35] C.M. Natarajan, M.G. Tanner and R.H. Hadfield, Supercond. Sci. Technol 25, 063001 (2012) arXiv:1204.5560. 